\title{
Statistical 3D Shape-Model Guided Segmentation of Cardiac Images
}

\author{
Y Shang ${ }^{1,2}$, O Dössel ${ }^{1}$ \\ ${ }^{1}$ Institute of Biomedical Engineering, University of Karlsruhe, Germany \\ ${ }^{2}$ Department of Electronic Engineering, Tsinghua University, P.R. China
}

\begin{abstract}
A novel scheme for the segmentation of 4-D MR cardiac images has been introduced in this paper. 3-D spatially hierarchical expressions of the statistical shape models for the cardiac chambers are constructed through principal component analysis (PCA) of the manually segmented training set. The difficult problem of landmarking before PCA is effectively solved through gradient vector flow guided $3 D$ active surface method. The hierarchical shape models are then used as constraint forces in the segmentation process. The system has been tested on 3D MR cardiac images. The segmentation results of the presented system are better compared with the active surface method, and are comparative to the manual segmentation results.
\end{abstract}

\section{Introduction}

Cardiac image segmentation is the prerequisite for constructing a realistic anatomical model of the heart, from which diagnostic information can be directly derived. Cardiac anatomical models are also the basis for further electro-physiological or electro-mechanical simulations. Although advances in medical imaging techniques enable us nowadays to get spatiotemporal cardiac images with ever-increasing quality, automatic segmentation still remains a difficult task because of the unavoidable presence of the imaging noise and the intricate structure of the heart itself. Among automatic image segmentation methods, deformable model method is the most promising one. Compared with traditional low level segmentation methods like thresholding, region growing, watershed, which are solely based on the information offered by the images, deformable model method utilizes prior shape knowledge to introduce constraints on the segmentation process [1,2]. Under the assumption of a general elastic body, deformable model methods fail to deliver satisfying results in some noisy and complicated cases due to undesirable deformation. To overcome this shortcoming, Cootes et.al. has proposed Active Shape Model (ASM) [3]. In ASM, a point distribution model derived through principal component analysis is used to capture shape variance from a training set, the following model deformation is then restricted within the variance space defined by this statistic shape model. The active shape model method offers an attractive way to deal with the difficulty of cardiac image segmentation, for it is natural to constrain the deformation of the heart into an allowable space.

Based on ASM, a framework for cardiac image segmentation is presented in this paper. The presented framework is novel in the sense of the spatial hierarchical expression of the statistical shape model, the landmarking method which combines the active surface method and the gradient vector flow image force, and the multistage segmentation. This paper is organized as follows: technical details of hierarchical shape model construction are given in Section 2. The multistage segmentation using the hierarchical shape model is presented in Section 3. Experimental results are illustrated in Section 4. In Section 5 the final summary and discussion are presented.

\section{Hierarchical shape model}

The frame work to construct the spatially hierarchical shape model is illustrated in Fig. 1. Hamarneh [4]

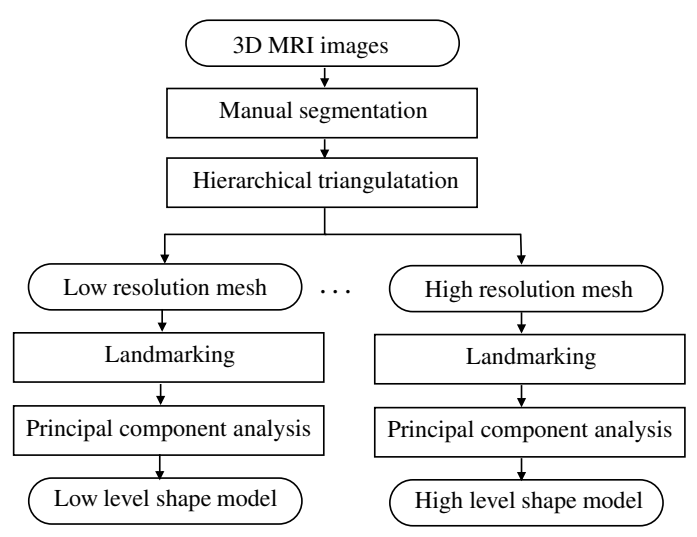

Figure 1. Procedure for constructing the hierarchical shape model. 


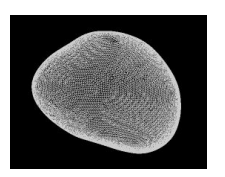

(a) (b)

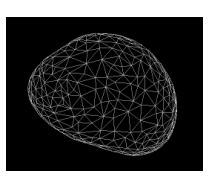

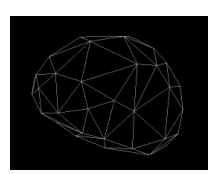

(c)
Figure 2. (a) the original triangle mesh created by marching cubes on manual segmentation result of left ventricle epicardium (23600 vertices), (b) after 50 decimation iterations (570 vertices), (c) after 500 decimation iterations (57 vertices).

proposed a hierarchical statistical shape model using PCA of the DCT coefficients of the spatial shape model, and Davatzikos [5] decomposed the shape model through wavelet transformation. Both of these methods include the forward and inverse transformations, and this results in high computational burden when large data sets are concerned. In comparison, our method is a multiresolution spatial representation without transformations. This is computationally efficient not only in the model construction phase but also in the segmentation phase.

\subsection{Hierarchical triangulation}

Hierarchical Triangulation is done on template images for different cardiac chambers which are constructed from the manual segmentation of the original 3D MRI images from the training set. The template image for each chamber is calculated as the average from the rigid aligned binary volumetric images. Triangulation is then performed on this template image using marching cubes technique, which results in triangle meshes with dense vertex distribution. The polygon reduction technique proposed in [6] is adopted to achieve hierarchical decimation of the initial triangle mesh. Aim of the decimation is to reduce the total number of triangles while preserving the topology and forming a good approximation to the original shape. The initial triangle mesh for left ventricle epicardium and the hierarchical decimation results are shown in Fig. 2.

\subsection{Landmarking using active surface with GVF}

Based on the hierarchical triangulation results on the template image, landmarking is the technique to establish the correspondence of the vertices on different triangle meshes segmented manually. The manually segmented triangle meshes are converted first into binary volumetric image, the landmarking is then performed between the template mesh and these volumetric images in the training set by a modified active surface method. This is closely related to the method proposed in [7], where the landmarking is carried out by deforming the template mesh to the volumetric image using energy minimization. In comparison, we have adopted the force equilibrium concept of active surface method. After alignment of the template mesh to the binary volumetric image by rigid transformation, the template mesh is deformed locally by the internal forces and the external GVF image force proposed in [8]. We have found that using GVF has greatly improved the performance of the landmarking over the traditional simple gradient image forces, for GVF creates much smoother and broader force fields from the binary images [9].

\subsection{Principal component analysis}

After landmarking of all the heart phases using the above mentioned deformable model method, Principal Component Analysis (PCA) is applied to the landmarked triangle meshes of the training dataset to derive an average shape and the allowable space of shape variation. The parameter space of the mesh model is greatly reduced by PCA. After PCA, model deformation is regulated by adjusting the reduced parameters only in the allowable space. As an example, the PCA results for left ventricular epicardium, endocardium, and right ventricular epicardium on a 4D cardiac data set are shown in Fig. 3. In this case, the manual segmentation results are landmarked with about 550 vertices. It is shown from the figures that the first four eigenmodes stand for more than $90 \%$ of the total shape variance of the training set. This means that only the few largest eigenmodes are needed in order to express other unknown shapes from the same anatomical class as that from the training set. Fig. 4 shows the first mode of variations for left ventricular endocardium at different resolutions.

\section{Multistage segmentation}

Aim of constructing the hierarchical shape models is to use them to regulate the surface deformation into the allowable variation spaces expressed by the shape models. Based on the hierarchical expression, the segmentation is divided into a multistage process. The first coarse stage regulates the surface deformation by the lowest level shape model with the lowest density of vertices to ensure a fast convergence to the primary stable state. The second stage is devoted to shape refinement which utilizes the finer shape models. The last stage is for the final finetuning through vertex relaxation. For the first two stages, the segmentation process is an iterative deformation and regulation circle until the stable state of the deformation is arrived. The last relaxation stage refines the surface 


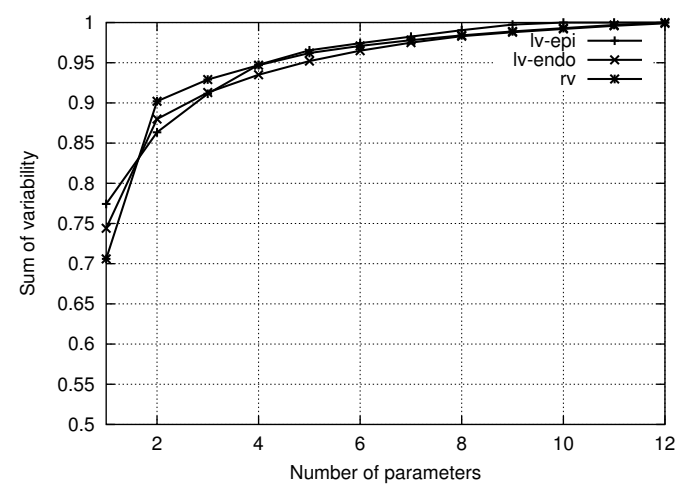

Figure 3. PCA results of manually segmented training sets (including left ventricle epi- and endocardium, right ventricle epicardium): the number of the eigenvectors and the corresponding variance captured by the eigenvectors.

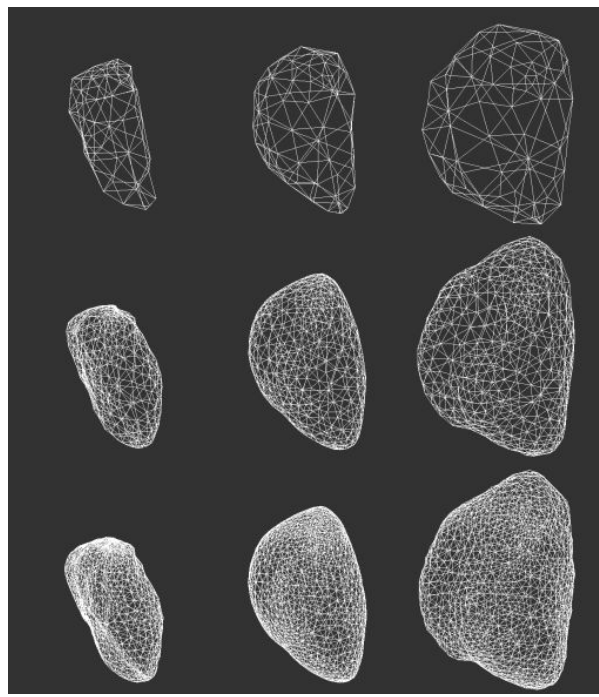

Figure 4. Variations corresponding to the largest eigenvalue of left ventricle endocardium at different resolutions (Vertices number 71, 555, 1685 respectively)

locally.

Regulation of the surface deformation is realized by first deforming the average shape of the PCA result, and then projecting the intermediate deformation result into the feature space introduced by the shape model. Suppose after PCA of the landmarked point distribution models from the training set, $\bar{S}$ is the average shape, the value of the $i t h$ element of the reconstructed shape vector $\tilde{s}$ formed by the intermediate deformation result $s$ is approximated by

$$
\tilde{s}_{i}=\bar{S}_{i}+\sum_{k=1}^{t} p^{k} V_{i}^{k}
$$

where $V_{i}^{k}$ is the ith element of the kth largest eigenvectors, $p^{k}$ is the $k t h$ reconstruction parameter and computed by

$$
p^{k}=\sum_{i=1}^{3 L}\left(s_{i}-\bar{S}_{i}\right) \cdot V_{i}^{k}
$$

In this stage, $p^{k}$ is truncated within the range $\pm 3 \sqrt{\lambda_{k}}$, for the variation lies in most applications within three standard deviations from the mean. The intermediate deformation result is reconstructed using Eq. (1), and it is used then as the initial shape for the following deformation. The whole process is terminated when the deformation of the surface and the change of the reconstruction parameters are within a certain threshold. After convergence on the coarse level, the result is landmarked to the finer level, and used as the initial shape. The total level of the shape model can be determined according to the applications.

In case the final result is over-regulated by the shape model, the final stage of the segmentation realizes the vertex relaxation using the above explained active surface method. The regulation using the statistical shape model is shut down in this stage, and the vertex deformation is guided by the local gradient information and is confined into a $3 \times 3 \times 3$ based neighbourhood region.

\section{Experiments}

To test the proposed segmentation system, we have used a 4D MRI cardiac data set offered by Philips Research Laboratories (Hamburg). This data set contains 25 3D cardiac frames. We have tested manual segmentation, active surface method with GVF, and active surface method regulated by the hierarchical statistical shape models on the left ventricle endocardium. The hierarchical shape models of the left ventricle endocardium is constructed using the 6th to 25 th frames from the total 25 frames. They are used then to regulate the segmentation process of the 1 st to 5 th frames which are not included in the training set. Fig. 5 shows the multistage segmentation results using three level statistical shape models for the left ventricle endocardium of the first frame. It is shown that the first coarse stage achieves only an approximation, which is then refined by the finer shape model. The result is better after vertex relaxation in the nearby neighbourhood. Fig. 6 shows the comparison between different methods. It is shown that the results of $3 \mathrm{D}$ active surface method are affected greatly by the image force field, and the surfaces are often attracted by false informations, while these shortcomings are overcome if the surface deformation is regulated within the allowable deformation space defined by the shape model. In this way a quality very similar to the manual method was achieved. 


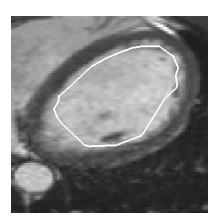

(a)

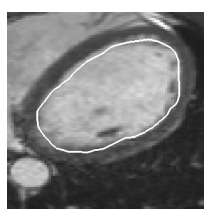

(b)

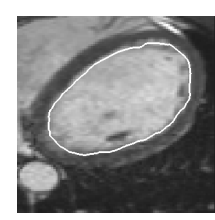

(c)
Figure 5. Results of multistage segmentation for the left ventricle endocardium of the first frame. For slice 32: (a) shape model with 71 vertices, (b) shape model with 555 vertices, (c) final relaxation result with 1685 vertices.

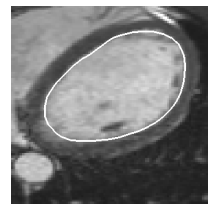

(a)

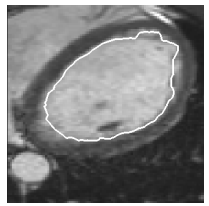

(b)

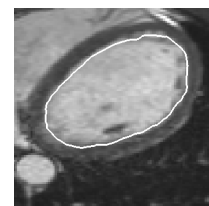

(c)
Figure 6. Comparison of the segmentation results for the left ventricle endocardium of first frame. For slice 32: (a) manual segmentation, (b) active surface method, (c) proposed method.

\section{Conclusions}

In this paper, we have presented a multi-stage active shape model based framework for cardiac data segmentation. The statistical shape models used in our system are derived through principal component analysis of the point distribution models landmarked using active surface method with a long range image force, GVF. The shape models are expressed in a spatially hierarchical way by different triangulation densities using the decimation technique. The advantage of the proposed system is that it integrates the manual segmentation, statistical shape model construction and multistage segmentation regulated by hierarchical shape models into a unified system, based on the same data structure, triangle meshes for the deformable surfaces. By this way, the computational burden of the system is low with robust and satisfying results even in ill-posed situations. For a 4D cardiac image data set with a high temporal resolution like the data set that has been used in our experiment, the segmentation results of the present frame can be used as the initial shape for the next frame.

Good results have been achieved using the presented method. More experiments and quantitative comparisons need to be carried out to test the proposed technique on images captured in different environments or through other modalities. A natural extension of this frame work is to construct a database for the hierarchical shape models, as soon as more segmentation results are derived using this method. Another research direction is multiple object segmentation where different cardiac chambers are segmented simultaneously considering the relationship between the chambers.

\section{Acknowledgements}

The first author is granted by DAAD (German Academic Exchange Service) for her PhD work. The authors would like to thank Dr. Manke and Dr. Stehning from Philips Research Laboratory in Hamburg for offering the 4D MRI data set.

\section{References}

[1] Kass M, Witkin A, Terzopoulos D. Snakes: Active contour models. Int J Comput Vision 1988;1(4):321-331.

[2] McInerney T, Terzopoulos D. Deformable models in medical image analysis: A survey. Med Imag Anal 1996;1(2):91108.

[3] Cootes TF, Taylor CJ, Cooper DH, Graham J. Active shape models-their training and application. Comp Vis Image Understand 1995;61(1):38-59.

[4] Hamarneh G, Gustavsson T. Combining snakes and active shape models for segmenting the human left ventricle in echocardiographic images. In Computers in Cardiology 2000. 2000; 115-118.

[5] Davatzikos C, Tao X, Shen D. Hierarchical active shape models, using the wavelet transform. IEEE Trans Med Imag March 2003;22:414-423.

[6] Schroeder W, Zarge J, Lorensen W. Decimation of triangle meshes. Computer Graphics SIGGRAPH 92 August 1992; 26(2):65-70.

[7] Kaus MR, Pekar V, Lorenz C, Truyen R, Lobregt S, Weese J. Automated 3-D PDM construction from segmented images using deformable models. IEEE Trans Med Imag August 2003;22:1005-1013.

[8] Xu C, Prince JL. Snakes, shapes, and gradient vector flow. IEEE Trans Med Imag March 1998;7:359-369.

[9] Shang Y, Dössel O. 3D active surface method for segmentation of medical image data: Assessment of different image forces. In Biomedizinische Technik, volume 48-1. 2003; 28-29.

Address for correspondence:

Yan Shang

Universität Karlsruhe, Institut für Biomedizinische Technik, Kaiserstrasse 12, 76128, Karlsruhe, Germany

Yan.Shang@ibt.uni-karlsruhe.de 